Slawomir Solecki, Department of Mathematics, Indiana University,

Bloomington, IN 47405. e-mail: ssolecki@indiana.edu

\title{
MAKING BOREL FUNCTIONS LOOK CONTINUOUS
}

\begin{abstract}
We investigate the possibility of making all functions in Baire class $\alpha, \alpha<\omega_{1}$, close to continuous by adding to them a single function of Baire class $\alpha+1$.
\end{abstract}

Remnants of continuity are preserved, in various senses, in arbitrary Borel functions. Yet another way in which this is true has been considered recently by several authors. Given $\alpha<\omega_{1}$, can we find a single function $f$ which makes all functions of the form $f+g$, with $g$ Baire class $\alpha$, look continuous? Obviously, we would like $f$ to be as simple as possible and the functions $f+g$ as close to continuous as possible. The following four definitions are normally used, in this context, to measure the "degree of continuity" of $f+g$. How to measure simplicity of $f$ will be clear from the statements of results. Let $g$ be a function mapping a separable metric space $X$ into the reals.

$g$ is Darboux if images of connected sets are connected.

$g$ is connectivity if the graph of the restriction of $g$ to any connected subset of $X$ is a connected subset of $X \times \mathbb{R}$.

$g$ is almost continuous if any open subset of $X \times \mathbb{R}$ containing the graph of $g$ contains also the graph of a continuous function from $X$ to $\mathbb{R}$.

$g$ is extendable connectivity if $g(x)=G(x, 0)$, for all $x \in X$, for some connectivity function $G: X \times[0,1] \rightarrow \mathbb{R}$.

It is known that if $X=\mathbb{R}$, then the above classes are getting properly smaller as we go down the list and that all of them contain the continuous functions.

Let us also fix notation concerning Borel functions. By $\mathbf{B}_{\alpha}, \alpha<\omega_{1}$, we denote the class of all functions $g: X \rightarrow \mathbb{R}, X$ separable metric, for which preimages of open sets are in $\Sigma_{1+\alpha}^{0}$ if $\alpha<\omega$, and in $\Sigma_{\alpha}^{0}$ if $\alpha \geq \omega$, where $\Sigma_{\alpha}^{0}$ is the $\alpha$ 'th class of the Borel hierarchy. (Remember that the enumeration of

Key Words: Baire classes, Borel functions, Darboux functions.

Mathematical Reviews subject classification: 03E15, 26A15, 26A21

Received by the editors July 24, 1998

* Research supported by NSF grant DMS 9803676 
$\Sigma_{\alpha}^{0}$ 's starts with $\alpha=1$ while that of the $\mathbf{B}_{\alpha}$ 's with $\alpha=0$.) In particular, $\mathbf{B}_{0}$ is the class of continuous functions, $\mathbf{B}_{1}$ the class of Baire class 1 functions, etc. In general, by a theorem of Lebesgue and Hausdorff, functions of Baire rank $\alpha$ coincide with $\mathbf{B}_{\alpha}$, for $\alpha<\omega$, and $\mathbf{B}_{\alpha+1}$, for $\alpha \geq \omega$. Clearly, $\bigcup_{\alpha<\omega_{1}} \mathbf{B}_{\alpha}$ is the class of all Borel functions. By $\mathbf{B}_{\alpha}(X)$ I will denote all functions in $\mathbf{B}_{\alpha}$ defined on $X$. For more details on the hierarchies of Borel functions and Borel sets see [K, Chapters 11 and 24].

It follows from the work of Ciesielski and Recław [CR], building on earlier papers by Fast, Kellum, and Ciesielski and Miller, that there exists $f: \mathbb{R} \rightarrow \mathbb{R}$ such that all functions in $f+\bigcup_{\alpha<\omega_{1}} \mathbf{B}_{\alpha}(\mathbb{R})$ are extendable connectivity functions. Such an $f$ clearly cannot be Borel. Later, Natkaniec and Recław [NR] showed that given $\alpha<\omega_{1}$, one can find $f \in \mathbf{B}_{\alpha+2}(\mathbb{R})$ for which all functions in $f+\mathbf{B}_{\alpha}(\mathbb{R})$ are Darboux, and one can find a Borel $f$ (without any control over its class) with all functions in $f+\mathbf{B}_{\alpha}(\mathbb{R})$ almost continuous. Below, answering a question from $[\mathrm{NR}]$, I prove a natural common generalization to the two Natkaniec-Recław results. As in the Natkaniec-Recław paper, the method of Cichon-Morayne universal functions is used. There is a new ingredient, however. It turns out that "strongly universal," in an appropriate sense, rather than universal functions are needed. I obtain their existence by a Kleene Recursion Theorem argument. Moreover, since tight control on complexity of the functions is needed, I employ in the proof the existence of a perfect set independent over $\mathbb{Q}$.

Theorem. For any $1 \leq \alpha<\omega_{1}$ there exists $f: \mathbb{R} \rightarrow \mathbb{R}$ in $\mathbf{B}_{\alpha}$ such that $f+g$ is an extendable connectivity function for any $g: \mathbb{R} \rightarrow \mathbb{R}$ in $\bigcup_{\gamma<\alpha} \mathbf{B}_{\gamma}$.

The following lemma is due to Cichon and Morayne [CM]. Recall that by $\underline{\mathbf{B}}_{\alpha}$ we denote the class of functions defined on separable metric spaces for which the preimages of half lines $(r, \infty), r \in \mathbb{R}$, are in $\Sigma_{1+\alpha}^{0}$ if $\alpha<\omega$, and $\Sigma_{\alpha}^{0}$ if $\alpha \geq \omega$. It is easy to see that $\mathbf{B}_{\alpha} \subseteq \underline{\mathbf{B}}_{\alpha} \subseteq \mathbf{B}_{\alpha+1}$. And again by $\underline{\mathbf{B}}_{\alpha}(X)$ I will denote the family of all functions in $\underline{\mathbf{B}}_{\alpha}$ which are defined on $X$. For a separable metric space $X$, a function $F: 2^{\mathbb{N}} \times X \rightarrow \mathbb{R}$ is called $2^{\mathbb{N}}$-universal for a class of functions defined on $X$ if this class consists precisely of all the functions $X \ni x \mapsto F(a, x)$ for $a \in 2^{\mathbb{N}}$.

Lemma 1. (Cichon-Morayne [CM]) Let $X$ be a separable metric space. For any $\alpha<\omega_{1}$ there exists a function from $2^{\mathbb{N}} \times X$ to $[0,1]$ in $\underline{\mathbf{B}}_{\alpha}$ which is $2^{\mathbb{N}}$-universal for $\left\{f: X \rightarrow[0,1]: f \in \underline{\mathbf{B}}_{\alpha}(X)\right\}$.

Note that, by a diagonal argument, Lemma 1 fails if the functions considered in it have $\mathbb{R}$ rather than $[0,1]$ as the target space. Similarly, as follows from [CMPS, Theorem 2.1], it fails if we replace $\underline{\mathbf{B}}_{\alpha}(X)$ by $\mathbf{B}_{\alpha}(X)$ regardless 
of whether we keep $[0,1]$ as the target space or replace it by $\mathbb{R}$. However, below we have a lemma, a variation of Kleene's Recursion Theorem similar to that in $[\mathrm{K}, 35.26]$, which shows that it is possible to find a function in $\mathbf{B}_{\alpha}$ that is, in the sense made precise by the lemma, more than $2^{\mathbb{N}}$-universal for $\bigcup_{\gamma<\alpha} \mathbf{B}_{\gamma}(X)$, for separable metric $X$, even with the target space $\mathbb{R}$. I leave it to the reader to see that a function $F$ with the property as in the lemma below is $2^{\mathbb{N}}$-universal for $\bigcup_{\gamma<\alpha} \mathbf{B}_{\gamma}(X)$.

Lemma 2. Let $X$ be separable metric. Given $1 \leq \alpha<\omega_{1}$ there exists $F$ : $2^{\mathbb{N}} \times X \rightarrow \mathbb{R}$ in $\mathbf{B}_{\alpha}$ such that for any $f: 2^{\mathbb{N}} \times X \rightarrow \mathbb{R}$ in $\bigcup_{\gamma<\alpha} \mathbf{B}_{\gamma}$ there exists $a \in 2^{\mathbb{N}}$ such that $F|\{a\} \times X=f|\{a\} \times X$.

Proof. Claim 1. For any separable metric space $Y$ there exists $G$ in $\mathbf{B}_{\alpha}$ that is $2^{\mathbb{N}}$-universal for $\bigcup_{\gamma<\alpha} \mathbf{B}_{\gamma}(Y)$.

Proof of Claim 1. Assume first that $\alpha$ is a successor, so $\alpha=\beta+1$ for some $\beta$. Let $F_{1}: 2^{\mathbb{N}} \times Y \rightarrow[0,1]$ be in $\underline{\mathbf{B}}_{\beta}$ and $2^{\mathbb{N}}$-universal for $\{f: Y \rightarrow$ $\left.[0,1]: f \in \underline{\mathbf{B}}_{\beta}\right\}$. Such an $F_{1}$ exists by the Cichoń-Morayne Lemma above. Let $A=F_{1}^{-1}(0)$. Define $F_{2}: 2^{\mathbb{N}} \times Y \rightarrow[1, \infty)$ by

$$
F_{2}=\frac{1}{F_{1}} \text { on }\left(2^{\mathbb{N}} \times Y\right) \backslash A \text { and } F_{2} \equiv 1 \text { on } A \text {. }
$$

It is easy to check that $F_{2} \in \mathbf{B}_{\alpha}$ and that for any $f: Y \rightarrow[1, \infty)$ in $\mathbf{B}_{\beta}$, for some $a \in 2^{\mathbb{N}}, F_{2}(a, x)=f(x)$ for all $x \in Y$. Define $G:\left(2^{\mathbb{N}} \times 2^{\mathbb{N}}\right) \times Y \rightarrow \mathbb{R}$ by

$$
G(a, b, x)=F_{2}(a, x)-F_{2}(b, x) .
$$

Clearly $G$ is in $\mathbf{B}_{\alpha}$ and it is $2^{\mathbb{N}} \times 2^{\mathbb{N}}$-universal for $\mathbf{B}_{\beta}(Y)$. Since $2^{\mathbb{N}} \times 2^{\mathbb{N}}$ is homeomorphic to $2^{\mathbb{N}}$, we are done.

If $\alpha$ is limit, fix $\alpha_{n} \rightarrow \alpha, \alpha_{n}<\alpha$. Let $F_{n}$ be in $\mathbf{B}_{\alpha_{n}+1}$ and $2^{\mathbb{N}}$-universal for $\mathbf{B}_{\alpha_{n}}(X)$ which exist by the argument in the previous paragraph. Divide $2^{\mathbb{N}} \backslash\{(0,0,0, \ldots)\}$ into infinitely many clopen sets $U_{n}, n \in \mathbb{N}$. Let $F \mid U_{n} \times Y=$ $F_{n} \circ \phi_{n}$ where $\phi_{n}=\psi_{n} \times$ id and $\psi_{n}: U_{n} \rightarrow 2^{\mathbb{N}}$ is a homeomorphism. Finally put $F \mid\{(0,0,0, \ldots)\} \times Y \equiv 0$. This $F$ works for $\alpha$. The claim is proved.

Let $\langle\rangle:, 2^{\mathbb{N}} \times 2^{\mathbb{N}} \rightarrow 2^{\mathbb{N}}$ be defined by

$$
c=\langle b, a\rangle \text { iff } c_{2 n}=b_{n} \text { and } c_{2 n+1}=a_{n} \text { for all } n \in \mathbb{N} .
$$

Obviously, $\langle$,$\rangle is a homeomorphism. The following property of the mapping$ will be crucial.

Claim 2. For any $b \in 2^{\mathbb{N}}$ there exists $a \in 2^{\mathbb{N}}$ with $\langle b, a\rangle=a$.

Proof of Claim 2. Consider the metric $\rho(c, d)=\sum_{n=0}^{\infty} 2^{-n}\left|c_{n}-d_{n}\right|$ for $c, d \in 2^{\mathbb{N}}$. As is well known $\rho$ is a complete, actually compact, metric on 
$2^{\mathbb{N}}$. As is easily seen $\rho\left(\left\langle b, a^{1}\right\rangle,\left\langle b, a^{2}\right\rangle\right) \leq \frac{1}{2} \rho\left(a^{1}, a^{2}\right)$. Thus, by the Banach contraction principle, for any $b$ the mapping $2^{\mathbb{N}} \ni a \mapsto\langle b, a\rangle \in 2^{\mathbb{N}}$ has a (unique) fixed point which proves the claim. (Obviously a direct proof of the claim, which is really a repetition of Banach's argument, is possible.)

Let $G$ be as in Claim 1 for $Y=2^{\mathbb{N}} \times X$. Now define $F: 2^{\mathbb{N}} \times X \rightarrow \mathbb{R}$ by

$$
F(\langle b, a\rangle, x)=G(b, a, x) .
$$

Since $\langle$,$\rangle is a homeomorphism, F$ is well defined on $2^{\mathbb{N}}$ and $F \in \mathbf{B}_{\alpha}$. Now, let $f: 2^{\mathbb{N}} \times X \rightarrow \mathbb{R}$ be in $\bigcup_{\gamma<\alpha} \mathbf{B}_{\gamma}$. Find $b_{0} \in 2^{\mathbb{N}}$ such that for all $(a, x) \in 2^{\mathbb{N}} \times X$, $G\left(b_{0}, a, x\right)=f(a, x)$. By Claim 2 find $a_{0} \in 2^{\mathbb{N}}$ with $\left\langle b_{0}, a_{0}\right\rangle=a_{0}$. Then, for any $x \in X$,

$$
F\left(a_{0}, x\right)=F\left(\left\langle b_{0}, a_{0}\right\rangle, x\right)=G\left(b_{0}, a_{0}, x\right)=f\left(a_{0}, x\right) .
$$

The following lemma is taken from $[\mathrm{R}]$ and $[\mathrm{CR}]$. Actually, it is not proved there that the function constructed is in $\mathbf{B}_{2}$ but, as already noted in [NR], it is straightforward to verify it.

Lemma 3. (Ciesielski-Rectaw $[C R]$, Rosen $[R]$ ) There exists a $\mathbf{B}_{2}$ function $f: \mathbb{R} \rightarrow \mathbb{R}$ and an $F_{\sigma}$ meager set $H_{1} \subseteq \mathbb{R}$ such that any extension of $f \mid H_{1}$ is extendable connectivity.

The next lemma is an old result due to Alexandroff and Urysohn [AU].

Lemma 4. (Alexandroff-Urysohn [AU]) Let $H_{1}, H_{2} \subseteq \mathbb{R}$ be $F_{\sigma}$ meager sets such that for any non-empty open $U \subseteq \mathbb{R}, H_{i} \cap U$ is uncountable, $i=1,2$. Then there exists a homeomorphism $\phi: \mathbb{R} \rightarrow \mathbb{R}$ with $\phi\left[H_{1}\right]=H_{2}$.

Proof of Theorem. For $\alpha=1$ the theorem is clear since $\mathbf{B}_{0}=$ the continuous functions. So assume that $\alpha \geq 2$. Let $P$ be a Cantor set independent over $\mathbb{Q}$ with $1 \in P$. Such a set was first constructed by von Neumann in [vN]. Let $P_{0}, P_{1} \subseteq P$ be two disjoint Cantor sets. Let $H=\mathbb{Q}+P_{1}$. Note that $F$ is a meager $F_{\sigma}$ and that $U \cap F$ is uncountable for any non-empty open $U \subseteq \mathbb{R}$. Fix a homeomorphism $\psi: 2^{\mathbb{N}} \rightarrow P_{0}$. Define $\psi^{\prime}: 2^{\mathbb{N}} \times H \rightarrow P_{0}+H$ by $\psi^{\prime}(a, x)=\psi(a)+x$. Note that $\psi^{\prime}$ is a homeomorphism. Continuity and surjectivity are obvious while injectivity and continuity of $\left(\psi^{\prime}\right)^{-1}$ follow from compactness of $P_{0}$ and $\mathbb{Q}$-independence or, more precisely, from the fact that any $z \in P_{0}+H$ can be uniquely represented as $z=a+x$ with $a \in P_{0}$ and $x \in H$. Also define $\pi: P_{0}+H \rightarrow H$ by letting $\pi=\pi_{1} \circ\left(\psi^{\prime}\right)^{-1}$ where $\pi_{1}: 2^{\mathbb{N}} \times H \rightarrow H$ is the projection onto $H$. Clearly $\pi$ is continuous.

Let $f: \mathbb{R} \rightarrow \mathbb{R}$ and $H_{1}$ be as in Lemma 3 . We can assume, by making $H_{1}$ larger if necessary, that for any $U \subseteq \mathbb{R}$ open nonempty, $H_{1} \cap U$ is uncountable. 
(One can easily see that any $H_{1}$ from Lemma 3 must have this property, so it is never necessary to enlarge it.) Now Lemma 4 allows us to fix a homeomorphism $\phi: \mathbb{R} \rightarrow \mathbb{R}$ with $\phi[H]=H_{1}$.

Now define $f_{1}: \mathbb{R} \rightarrow \mathbb{R}$ by letting $f_{1}$ be equal to $f \circ \phi \circ \pi$ on $P_{0}+H$ and 0 outside $P_{0}+H$. Since $\phi$ and $\pi$ are continuous, $f_{1} \mid P_{0}+H$ is $\mathbf{B}_{2}$ on $P_{0}+H$. Since $P_{0}+H$ is $F_{\sigma}, f_{1}$ is $\mathbf{B}_{2}$ on $\mathbb{R}$.

Let $F: 2^{\mathbb{N}} \times H \rightarrow \mathbb{R}$ in $\mathbf{B}_{\alpha}$ be as in Lemma 2 for $X=H$. Define $f_{2}: \mathbb{R} \rightarrow \mathbb{R}$ by letting it be $F \circ\left(\psi^{\prime}\right)^{-1}$ on $P_{0}+H$ and 0 on $\mathbb{R} \backslash\left(P_{0}+H\right)$. By an argument as for $f_{1}$ we get that $f_{2}$ is $B_{\alpha}$.

Define $k=f_{1}-f_{2}$. We claim that for any $g: \mathbb{R} \rightarrow \mathbb{R}$ in $\bigcup_{\gamma<\alpha} \mathbf{B}_{\gamma}, k+g$ is extendable connectivity. Fix such a $g$. Then for some $a \in 2^{\mathbb{N}}, F \mid\{a\} \times H=$ $\left(g \circ \psi^{\prime}\right) \mid\{a\} \times H$. It follows that

$$
f_{2}\left|\psi^{\prime}[\{a\} \times H]=F \circ\left(\psi^{\prime}\right)^{-1}\right| \psi^{\prime}[\{a\} \times H]=g \mid \psi^{\prime}[\{a\} \times H] .
$$

Now since $k+g=f_{1}+\left(g-f_{2}\right)$, we get

$$
(k+g)|\psi(a)+H=(k+g)| \psi^{\prime}[\{a\} \times H]=f_{1} \mid \psi^{\prime}[\{a\} \times H] .
$$

By unraveling the definition of $f_{1}$, we get that for any $x \in H,(k+g)(\psi(a)+$ $x)=f \circ \phi(x)$. It follows that any extension of $(k+g) \mid \psi(a)+H$ can be obtained from an extension of $f\left|H_{1}=f\right| \phi[H]$ by precomposing with a homeomorphism of $\mathbb{R}$ (namely $\mathbb{R} \ni x \mapsto \phi(x-\psi(a)) \in \mathbb{R}$ ); thus, it must be extendable connectivity. It follows that $k+g$, being such an extension, is an extendable connectivity function.

\section{References}

[AU] P. Alexandroff, P. Urysohn, Über nulldimensionale Punktenmengen, Math. Ann., 98 (1928), 89-106.

[CM] J. Cichoń, M. Morayne, Universal functions and generalized classes of functions, Proc. Amer. Math., 102 (1988), 83-89.

[CMPS] J. Cichoń, M. Morayne, J. Pawlikowski, S. Solecki, Decomposing Baire functions, J. Symb. Logic, 56 (1991), 1273-1283.

[CR] K. Ciesielski, I. Recław, Cardinal invariants concerning extendable and peripherally continuous functions, Real Analysis Exchange 21 (1995/6), 459-472.

[K] A. S. Kechris, Classical Descriptive Set Theory, Springer-Verlag, New York, 1995. 
[NR] T. Natkaniec, I. Recław, Universal summands for families of measurable functions, Acta Sci. Math. (Szeged), 64 (1998), 463-471.

[vN] J. von Neumann, Ein System algebraisch unabhangiger Zahlen, Math. Ann., 99 (1928), 134-141.

[R] H. Rosen, Every real function is the sum of two extendable connectivity functions, Real Analysis Exchange, 21 (1995/6), 299-303. 